\title{
Revitalising and enhancing sewing skills and expertise
}

Critical skill gaps highlighted by UK professionals in the fashion industry and identified by Government bodies were evidenced in the classroom while observing fashion students. The issues include lacking an understanding of garment construction quality, technical knowledge and skills, and timescale boundaries. It was also observed that students lack the confidence to experiment with construction techniques. A study was initiated to revitalise and enhance sewing skill capability through a purposely designed resource tool. Secondary research and interview responses from industry personnel working closely with undergraduate students and graduate designers substantiated the preliminary observations. The resource tool samples were inspired by two UK archive collections enabling the inclusion of lesser-used complex construction techniques. The tool is described, its effectiveness discussed and future developments are given. When fully developed, the resource tool could also enable craft dressmakers to develop and enhance their skills and expertise, by introducing them to less-documented techniques.

Keywords: skill; sewing; design; technical; seam; tool

\section{Introduction}

In the UK, there is a general assumption that fashion students beginning their degree will have basic sewing skills and knowledge gained from previous study or experience. Students will then develop design skills and further technical knowledge that ensures they are suitably prepared for a career in the fashion and clothing industry. However, it is increasingly evident from industry personnel and educators that this is rarely the case which. This is supported by academic literature and the UK government and commercial reports. Observations in the classroom also have also-revealed that students often demonstrate theira lack of understanding of the importance of garment construction quality for different market sectors. In addition, the observations reveal an inability to identify appropriate manufacturing and finishing techniques for set budgets. While design and cut skills have been seen to take precedence, construction methods, which are explored through sampling, design development and garment realisation, are 
often perceived by students as being uninspiring and rudimentary. As a result, students' work often lacks experimental enquiry. Furthermore, some students seem to lack confidence, or are otherwise reluctant to explore innovative construction techniques.

It is considered that the identified skills gap, or lack of sewing skills acquisition and expertise, is likely to be a result of changes to school education priorities and to changes in societal attitudes. Such changes have essentially demeaned the value of sewing skills, and thus relegated the activity of dressmaking to lower-class domesticity orders. However, basic dressmaking skills are at the forefront of garment construction and garment design for commerce. In addition, the craft of dressmaking purely as a hobby is also well deserving of consideration in the context of revitalising sewing skills, knowledge and expertise. While there is a growing interest in the craft, particularly in the UK, that is now well supported by popular TV programmes, resources for developing and enhancing sewing skills at high levels of expertise are limited.

A resource tool was developed in order to educate and inspire fashion design students in an attempt to address the skill gap. The tool provides an aid to revitalise and enhance garment construction knowledge, skills and expertise through guidance and instruction. The resource samples were inspired by clothing collections held in two UK archives. This enabled the revitalisation of some of the more complex construction techniques that are no longer commonly used in contemporary mainstream fashion. The resource tool briefly comprises seam, pleat and fastening types, which were designed to provide basic sewing techniques that engage the user and promotes experimentation with more advanced applications. The resource tool is described in this paper and its effectiveness is discussed. The paper concludes with suggestions for future developments.

While the tool was developed and tested with fashion students in the context of Higher 
Education in order to address the problem specifically for the industry sector, it is also considered to have further uses. When fully developed it could serve as a useful tool for craft dressmakers to help them to develop and enhance their skills and expertise, and to introduce them to less documented techniques. Therefore, it could potentially extend the revitalisation of sewing skills beyond academia.

\section{The skills gap}

According to Romeo \& Lee (2013, p. 13), found the lack of garment construction skills among creative designers is a to be one of the major concerns forfrom fashion industry personnel personnel, which were cited as one of the most frequently lacking skill areas for creative designers. They proposed that Poor knowledge of seams and construction was suggested to be-a leading factor in preventing designers from translating creative ideas into a-garments that are suitable for ean be-manufacture $\underline{\text { is their lack of }}$ knowledge of seam types and construction methods.

Jamie Petrie, manager of the fashion and textiles sector at skills agency Skillset, suggests that there is an oversupply of design graduates without the technical, operations and manufacturing skills to meet the supply and demand of the clothing industry (Drapers, 2011). This supports the concept that design creativity alone leaves students ill-equipped for industry, as they lack the skills and knowledge needed to put orders into production.

Hayes et al. (2012, p. 48) make specific distinctions between the designer and technologist. They suggest that a designer has 'the flair to innovate and create new product designs' while production technologists maintain 'design integrity, whilst being a realist in developing the design into mass production'. 
Swift and Brown (2003, p. 827) discuss product over-design as being a result of the gap between design knowledge and manufacturing skill. They found that 50 per cent of product development was wasted through the rework of over-complex design, which often amassed substantial financial implications. They propose clearer communication of manufacturing knowledge during the early stages of design. This would allow problems of over-complexity to be resolved more efficiently before the manufacturing stage begins.

Furthermore, O'Driscoll (2002, p. 318) states that many occasions occur in the industry where poorly performed production processes result from an insufficient understanding of production capabilities linked to design requirements. O’Driscoll (2002) suggests that this occurs due to the translation of design details into manufacturing processes often being inadequately explained. He recommended that the designer and manufacturer should have some significant overlap in knowledge of design aesthetics and technical competency to address this.

\section{Decline in needlecraft in schools}

Frayling (2011, p.11) discusses considerable anecdotal evidence that higher education design students are demanding more hands-on activities. This could be in response to the decline of such education in schools. The decline of needlecraft in secondary school education is suggested to be a significant factor relating to the skill decline, and may be a result of today's throw-away culture (Norum 2008, p. 125).

Norum (2008) proposed current clothing consumption practices as being accountable in the lack of sewing skills of millennial's. Norum's study examined millennials' clothing maintenance skills and practices, and indicated that there was little evidence of repair work undertaken as a regular activity. It was also established that 
most repair work carried out involved minor skilled tasks such as 'sewing on buttons and fixing hems'. The survey indicated that many participants did not have the necessary skills to repair clothes and that an overall decline in repair skills was in part due to a decline in teaching the skills in schools. It is also recognised that such manual skills demand time and patience to master. The 2013 Education Manifesto for Craft and Making realised a drop of 25\% in craft-related GCSE engagement (2007-2013). This was due to concerns among parents and children that such time-consuming subjects may detract from academic performance in other subjects (Crossick \& Greenlees, 2014, p. 5).

In line with the production of a sewing skills resource tool, the value of sewing from an academic perspective has been considered. The significance of teaching sewing skills withinen fashion design courses seems to be downplayed from certain academic viewpoints. Beard and Slocum (2005, p. 299) acknowledge Buckland's (2000) paper on the history of sewing, which states its importance as controversial. Yet some sewing competency is recognised as enhancing students' understanding of garment structure to execute projects with informed judgement.

McRobbie (1998, p. 57) also discusses a marginalisation and downgrading of the practical skills involved in making clothing. She suggests that as an academic subject, fashion educators hasve remained apprehensive to acknowledge the value of technical skills. There is also an interesting historical viewpoint around the successful status of fashion as a creative artistic subject disassociating itself from the menial skills of dressmaking. This seems to emphasize the division of labour between design and sewing.

\section{The sewing resource tool}


As previously stated, the aim of the resource tool iwas to help students to better understand how to link design and construction skills together,- with $t$ The ultimate intention of the resource tool is to improveing their students' sewing skills. The tool consists of 33 different sewing techniques. To remain consistent, each sample type was A4 sized, titled and attached to an A3 card, which could be removed and handled easily. The reverse of each card featured an illustrated step-by-step guide to achieving the technique in four stages, as in the example (figure 1). The resources did not include written instruction as students often prefer a visual demonstration.

[Figure 1 here]

The samples awere categorised into high, medium or low skill levels and subcategorised into two distinct groups - creative techniques and technical techniques.

The creative range samples required students to use independent interpretation, while the technical sample range required students to remember previously taught techniques to perform stages in logical sequences. Table 1 categorises the specific technique types. Low skill techniques in the creative samples include: knotting, knotting $z$, faggoting / dissolvable, and pleating. Low skill techniques in the technical samples include: overlocking, 1emopen-seam top-stitched, hand sewn hem and pin hem. Medium skill techniques in the creative samples include: butt seam with strip, butt seam, vinyl hem, ridgeline hem and elastic seam edge. Medium skills techniques in the technical samples include: French seam inserted, French seam, lapped seam, run and fell seam, elastic seam overlocking, gathering lapped seam, alternative to darts and exposed tape zip fastening. The high skills techniques in the creative samples include: Hong Kong binding, French seam adapted, gathering 1, gathering 2 and drawstring. The high skills techniques in the technical samples include: double bindings, single binding, 
double binding hem, invisible zip, bagged-out zip with exposed teeth, rouleau loop fastening and continuous strip vent opening.

\section{[Table 1 here]}

\section{Methodology}

Figure 2 shows the stages of the research methods undertaken for the study.

[Figure 2 here]

-Following the secondary research phase, two archive visits were undertaken in order to draw inspiration for a suitable range of constructionelothing techniques for the resource tool. Two semi-structured 50 minute interviews were conducted with two London based SME (small to medium enterprise) manufacturers to develop a further understanding of the perceived skills gap debate. The interviews took place on location in order to experience the typical working environments. The three respondents included the two directors at manufacturer one, and the Business Director at manufacturer two. Key findings from the secondary research informed the twenty interview questions covering four topics; the skill gap between designers and industry standards; construction knowledge and awareness; the value of manual skills and production roles; and garment construction/finishing methods, plus factors that may lessen the skills gap. Following the interviews, audio files were transcribed.

A pilot study followed with sixty-two second year 13/14 BA (Hons) Fashion Design students, who were observed using a purposely created small-scale small-seale variationversion of the final resource tool, which consisted of ten different samples including; French seams, butt seam, lapped seams, binding variations, rouleau loops and spaghetti straps, faggoting and decorative finishes. - Second year students were selected 
for both the pilot study and the main experiment as this year group had a degree of experience and confidence in operating sewing machines. Also, tThey also-had an understanding of the essentials of fabric sampling and knowledge of the skills involved in constructing basic garments from the first year of their study.

During the pilot study students were observed using thea small selection of purposely created stitch and seam type resources. Students were required to produce a series of fabric samples using creative techniques and processes demonstrating their ability to recognise different manufacture techniques, fabrics and stitch types. The observations focused on the implementation of the resources to better understand their effectiveness. Field notes documented student sewing skills, and photographs were taken to record the observations. A further academic staff member witnessed the observations to acknowledge the validity of the field notes. Areas for improvement were identified through the observations. The pilot study samples were: French seams, butt seam, lapped seams, Hong Kong finish and other binding, rouleau loops and spaghetti straps, faggoting and decorative finishes.

A focus group followed with four of the students from the pilot study cohort.; €The audio files from the session were later transcribed. The purpose of the focus group was to gain constructive feedback to inform the design of the resource tool for the main experiment in addition to the data obtained from the archive visits, interviews, and pilot study.

Due to the timing of the pilot study and the main experiment two different second year groups were used as the pilot study participantsgreup were on work placement at the time of the main experiment. As comparisons in skill development could not be assessed it is recognised that the results may be limited at this stage. 


\section{The main experiment methodology}

The main experiment resource tool used in the main experiment comprised of 33samples. Fifty-six students were asked to create a series of sewing samples themed around different construction topics. The resource tool was divided into weekly thematic topics. The hanging samples were removed from a rail and placed on the tables in the studio. This ensured that the tool could be easily handled by the whole group. The fifty-six students were divided into two groups A and B. All students completed three hours of manufacture (including 90 minutes for the test) and three hours of patterncutting each week. Groups A and B rotated from manufacture to pattern-cutting in the morning and afternoon, in different sides of a screened studio, allowing the test to be managed effectively.

\section{[Table 2 here?]}

The fifty six students were divided into two groups; twenty eight in the morning for 3 hrs of sewing (including 1.5 hours for the test) and three hours for pattern-cutting. The groups rotated from sewing to patterns am/pm. The pattern group were at one side of a targe studio, the sewing were the other side. This ensured that the students were not all present in the session athe same time.Students were limited to the times that they could use the resources in the studio; 90 minutes each week (over three, three hourthreehour sessions). While using the resources, sThe students were observed and supervised at all times. After the experiment was complete, students were able to use the sewing resources as required, unsupervised and out of session times. Of the 56 students, only seventeen student samples were assessed following the main experiment as they were the only students to attend all three sessions in which the experiment was conducted, and to complete all of the subsequent questionnaires. However, $\mathrm{t}$ This did not hinder the remaining thirty-nine students as they were all expected to produce samples using the 
resources for their project work regardless. While the 17 students assessed were required to produce a minimum of three samples per session, some achieved up to seven or eight.

The students created seams, finishing and hem types in week one; pleats, folds and gather types in week two; and fastening technique types in week three. The students engaged with a set of two tests were developed-per weekly session. The first test for each weekly session was conducted before the resourcess__were introduced. and the students then engaged with the second test was conducted-while using the resource tool. This allowed the researcher to for the observeation any-of variations in the students' sampling before and after the resource tool implementation in order to make comparisons.

In the first test each week, students were asked to create a minimum of three examples around the themed areas using_calico fabric provided, withalongside the $\underline{\text { addition of and other trimshaberdashery items such as pins, tapes, elastics and threads. }}$ The three samples were collected for assessment purposes. Students were then introduced to the fabric resources which were relevant to each week's session topic. Time was taken to discuss each technique to the student group. During the second test students were asked to produce an additional three samples (minimum)-using the resource tool as inspiration. Both sets of samples_-were collected and assessed $\underline{\mathrm{u}} \mathrm{u}$ sing a scale of 1 (poor) to 5 (excellent) for against the following criteria:

(1) The quality of the samples produced

(2) The control and precision of the sewing

(3) The manipulation of the materials

(4) Dexterity in sample development

(5) The functionality of the samples 
(6) Inventiveness of techniques explored

(7) The appropriateness of the technique for the fabric

(8) The sophistication of the technique

(9) Creativity of the approach

(10) The aesthetic value of the sample.

Named plastic wallets were provided at the end of each test so participants could place their samples inside for the assessment. The samples from test one, week one were assessed individually using the above criteria. This was then repeated for test two. An overall collective total for each sample in test 1 was calculated. This was then repeated for test two. Both sets of results were then compared and analysed. This process was repeated each week.

The experiments were controlled in order to ensure the fairness and equality of the testing. Work produced by the same seventeen students during each testing period over three consecutive sessions was assessed. Students were not able to use electronic devices to access the internet and were only shown the fabric resources for guidance after the first set of samples had been completed and collected. At the end of each of the three sessions the students were issued questionnaires. The questions were linked to the featured weekly techniques to ascertain their thoughts and opinions on the implementation of the resource tool.

\section{Findings}

The semi-structured interviews with the clothing manufacturers focused on the problems encountered whilst working with undergraduates and recent graduates during the manufacturing of collection garments. The major issues identified were: 
(1) Generally limited technical knowledge including pattern cutting and construction awareness and ineffective communication skills to realise garments appropriately. These-which affects the flow of garment realisation, costing, time allocation and the depth of involvement required from the company. Also, nAot able to produce adequate pattern work or technical specification packs to support garment manufacture. This which contributes to higher costs and delays on production.

(2) Unrealistic expectations and viability of garment design - little understanding of the qualities of certain fabrics, construction methods or finishes which resulted in the re-working of designs and patterns incurring additional cost.

(3) Lack of financial knowledge and awareness (costing) - rated on a par with lacking technical knowledge. A concerning point surrounding the financial implications issue appeared to be based on design intervention. This was due to undergraduates and new graduate designers having poor knowledge of construction.

In discussing ways to combat the skills gap and recommendations for improvements, there was a positive response to the idea of exposing undergraduates to further techniques through the aid of a resource tool comprising stitch and seam types. It was also affirmed that sewing knowledge considerably enhances design ability.

The focus group students agreed that they would not have liked a more extensive range of resources and techniques as they preferred to use the resources as a stimulus for developing their own ideas. They did however suggest that a wider range of fabrics in the resource tool would have allowed for clear recognition of appropriate production techniques and finishes. A list of options for the presentation of the resource tool samples included; hanging on a rail, boxed, mounted on walls, integrated into a 
garment, or as digital images. During the focus group discussion, participants agreed

that the samples should be presented as hanging samples on a clothing rail. This ensured

that each technique could be easily removed and placed on tables, subsequently

allowing students to appreciate the handle and drape.

The students confirmed that they had gained very little understanding and appreciation for manufacturing in their first year of study, and had therefore assumed that amendments made to their designs by staff was in disregard of their creativity. Building on the confidence gained after teaching and practicing garment manufacture techniques, the undergraduates suggested that they were able to recognise the flaws of their designs more easily now and could better understand the feedback from staff, enabling them to more comprehensively consider the manufacture stages of their designs. They noted that it was pointless designing something that they could not visualise being made.

\section{Main experiment findings}

There were a $\underline{\mathrm{A}}$ number of issues were highlighted through the observations in relation to the main experiment. The most significant issue was the lack of independence to explore techniques without the aid of the Internet or the resource tool for inspiration. This highlighted the importance of the resource tool as a positive teaching aid. However, it, yet also suggested that students were not able to recall techniques demonstrated in their previous year of study. It was therefore This was-considered to be a very interesting factor which instigated further exploration into the reasons why, over the three-week testing stage, certain techniques were more or less appealing (discussed further in the following section). 
It was found that a large percentage of the student group did not understand $\underline{\text { some basic, fundamental seam classifications and types, such as the commonly used }}$ $\underline{1 \mathrm{~cm} \text { open seam. During the testing stage many students did not realise that a straight }}$ and curved variation of this seam (shown in figures $3 \& 4$ ) were essentially the same type.

It was found that a large percentage of the student group did not understand some of the basic seam classifications and types, such as the basic as variations of the

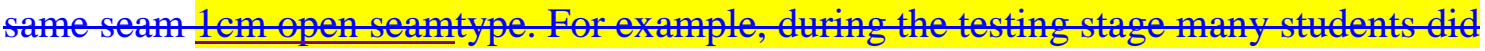
not realise that a curved straight and curved varitation of the $1 \mathrm{~cm}$ open seam (shown in figures $3 \& 4$ 3) was the same seam type as a straightwere essentially the same seam type, albeit one was curved. 1em open seam_(shown in figure 4).

[Figure 3 here]

[Figure 4 here]

\section{Questionnaire findings}

The series of questionnaires issued after each testing session gave students the opportunity to evaluate the techniques they had implemented. Each sample was analysed for its potential effectiveness and engagement. The results of the popularity of each of the 33 techniques, as rated by the seventeen students participating in the main experiment, are shown in table $\underline{2 t 3} 4$. The dark grey bars indicate the creative sample range and the light grey bars indicate the technical sample range.

\section{[Table $\underline{2+3}+$ here]}

The results in table $\underline{2 t 3} 4$ show the most popular techniques amongst the range of 33 samples. The most popular were generally from the creative technique group, and in 
particular the knotting samples (shown in figures 5 and 6), which were both valued as a low level skill technique for the experiment.

[Figure 5 here]

[Figure 6 here]

Of the two versions, the bias cut knotting sample was the most engaging technique, though this may have been due to the gingham fabric choice over calico. Twelve of the seventeen students stated that they had replicated this technique in some form during the experiment. The production of this technique involves very minimal skill and equipment in terms of sewing only requiring scissors to cut and snip into fabric and to join the strands together with a series of hand tied knots. The technique could be achieved very easily with minimum guidance or recall. The knotting instruction diagram was shown in figure 1.

From the results, it is fair to assume that in general the creative samples were more popular and engaging for students, perhaps due to the aesthetic values and appearance, and the relative ease in production of these techniques compared to the technical range.

Another popular technique was the faggoting dissolvable sample (shown in figure 7) which was valued as a lower skill creative technique. Similar to the knotting and gathering samples, this technique required an emphasis on more creative methods of stitching and not necessarily on recall. This particular sample required cold-water dissolvable film and stitching to link the fabrics together.

[Figure 7 here] 
From the results of this experiment there is a pattern to suggest that the more creative techniques, which require individual interpretation instead of recalling stages of a process, were the most popular for students to recreate. This may implywould suggest that in order for students to connect with more difficult technical samples there needs to be some kind of creative link to engage them in the process. Interestingly though, the rouleau loops fastening from the technical sample group (shown in figure 8) was rated third highest by the students, yet it was the second most highly skilled technique in the

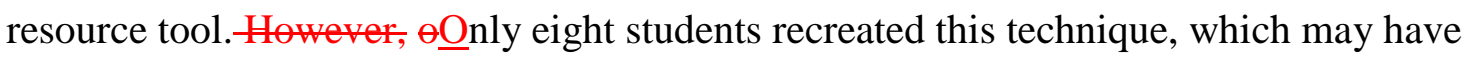
been due to the difficulty in achieving it accurately. This is similar to the findings for the binding samples where bias fabric lengths need to be pre-cut and the recall of the construction stages were necessary.

[Figure 8 here]

Further findings indicate that students generally avoided difficult tasks such as the cut and bag-through stage of the bias-cut strips to create the rouleau loops, which was effectively one of the most difficult stages of construction for this sample. The students leaned towards easier techniques, such as using a variety of adaptations including stay tapes, trims and types of elastic. Such actions demonstrate either an unwillingness to use the resources tool, or a lack of understanding of how to use the tool to recreate the resources shown. It does however demonstrate creative initiative and independence. Avoiding the difficult stages of some sample techniques however outweighed the effectiveness of the aim of the resource tool, as students did not fully engage with the essential stages required to recreate some of the fastening types.

Linked to the idea of students preferring the creativity in sampling as opposed to the more technically skilled versions, the binding samples were rated as the highest 
skilled of the resources. Again, tThis again-supports the idea of students preferring the creative techniques as they rated this sample rather unfavourably. This suggested that the difficulty in achieving this technique was off-putting as only six of the seventeen students replicated this finish. Also, the samples created in most of the student interpretations were poor in terms of accuracy. This may be due to the importance of recall for certain stages of construction, or due to the fact that further cutting of bias binding would be required. ItThis seems to indicate that students were in some way unwilling to use their own initiative to create adequate versions.

Of the 33 techniques introduced to the students over the three sessions there were some other interesting assessments. Students investigated ruching and gathering fabric with elastic trim, and results from the questionnaire findings suggest that these particular samples were highly engaging. Like the knotting samples discussed above, the elastic and gathering samples were intended as moderately simple techniques and produced quick, adjustable aesthetically creative finishes. During the observations, the majority of students did not understand how to use elastic effectively. The simple, basic process of stretching elastic and machine stitching it securely onto fabric was surprisingly challenging for the majority of students to replicate, which was noticeable in the assessment of students' samples.

The least favourable examples were the hand-sewn hem, overlocking types and the insertion of an invisible zip. These techniques all required some type of advanced technical skill often with little room for innovation or error in the finish. $\underline{\text { The dart and pleat sample, which featured a mock waist dart alongside a series of knife }}$ pleats (of the same width as the dart to illustrate equivalent features) required a good deal of skill to create. This sample helped students appreciate dart-equivalent seam lines or pleats; recognising that volume could be supressed by seam lines, pleating or 
gathering was generally notmn considered. The technique may have received a low rating because it required students to be fully aware of dart manipulation and suppression for reduction of volume in clothing prior to the experiment.

One of the least interesting engaging techniques for students was the invisible concealed zip. This was one of the most technical samples to achieve out of the full range of samples and it is not often that students can achieve a professional finish to this zip type without the assistance of technical support. The memory involved in recalling stages of the zip technique could be the main reason for the low rating. There is a pattern to suggest that use of memory and skill in recalling stages of a process, such as the zip insertion, binding, overlocking and the narrow, twice-turned pin hem, aptly named to illustrate its fine pin-width, relate to the less popular choices. At least for these students, the more creative easy to interpret samples such as the knotting and faggoting techniques appear to be the most popular.

\section{Significance of resources}

As students were asked to gauge their sewing ability before and after the experiments over the three sessions, there was a noted increase in ability levels from the intermediate to advanced bands only for a minority of students. However, nine of the seventeen students believed that they were already operating at an advanced sewing ability level before the resource implementation. Two students genuinely believed felt that their sewing abilities had been further advanced as a result of the resources. 
It was surprising to reveal that a large percentage of the group already believed that their sewing abilities were at an advanced level, as at the onset of the experiment the majority of second year students would more realistically be at an intermediate or basic level of ability. Being aware of the general standard of sewing work produced at a second-year intermediate level, it was considered if students were fully aware of the definitions of each sewing category. The simplistic categorisations of the four areas; including basic, intermediate, advanced and expert classifications may have benefited from further description to ensure students were able to categorise themselves accordingly. It may be suggested that the length of time in which students were given to experiment with the sampling was too limited to make a significant impact on their learning.

In reviewing the analysis of student sewing ability levels, it would seem that there is room for development in the style and detail of the resource tool. The length of $\underline{\text { time in which students were given to experiment with the samples may have been }}$ limited. The resource tool was a useful aid; however, it did not have a significant impact on their learning. Editing timescales and the complexity of each technique could allow for more significant results in further research development.

In reviewing the analysis of sewing ability levels, it would seem that there is room for further development in the style and detail of the resource tool. Also, the timescales and complexity of sampling could allow for more significant results in further research development.From the results, it was also anticipated that more students would have progressed on from a lower to a higher banding after the sampling implementation, yet this was not the case.

When combining the overall marks from the seventeen students over three weeks, in the final week of testing there was quite a large decline of overall marks in 
terms of quality of sampling. It appears that as the techniques in the sessions became more advanced, the results for each student were generally lower. In week one the collective results from the seventeen assessed student samples were considerably higher in comparison to weeks two and three. There are perhaps a few reasons for this; one is that students might have been more engaged in the testing as this was the first of three sessions of experimentation. Secondly, they may have found the resources in week one to be more successful in aiding their development. Thirdly, students may have felt more confident with the samples because they were all based around seam adaptations, which are the basic and fundamental foundations when learning how to sew.

It is worthwhile to note that the dramatic change in assessment results throughout weeks one, two and three of testing highlights the overall effectiveness of the range of samples introduced in each session. The results suggest that if further developments to the resource implementation were made, additional samples would need to be developed in fastening and construction techniques and in the use of darts, tucks and pleats in clothing to allow students to become more confident with these skills.

In order for this type of sample testing to become more successful as a learning resource, the timescales for its implementation into teaching, and the type and style of the selection of sampling, should be considered. This is perhaps due to both the timeframe and the resource type, to some extent, being limited as they did not appear to have a considerable impact on the advancement of the students' sewing ability. However, there were two students who noted an improvement in their ability as a consequence of the testing. It may have been that these two students were the most honest, or that the other students had inadvertently overestimated their earlier capability. 
For this reason, the sample assessments were more valuable for evaluating actual skills improvement.

\section{Conclusion and future development}

From observing and assessing students and their work over time, it was realised that students and graduates generally lack the sewing skills that are required by the fashion and clothing industry which initiated this study. Following a literature review, interviews with industry personnel and two archive visits, a resource tool was developed as an aid to revitalise and enhance garment construction knowledge, skills and expertise.

From the sampling results of the main experiment it is clear that there needs to be a strategy to engage students in this method to improve their learning. The timescale for the experiment did not allow for this level of skill acquisition. A major factor for consideration in any potential future development would be in introducing the resources at a much earlier stage of the course. General feedback during observations suggested that the implementation of resources would have been more beneficial to learning if integrated into year one, as the majority of students appreciated the value of the tool for their sewing knowledge development. To some extent the researcher was aware of this recommendation before and during this experiment, yet due to the limitations in teaching timescales in the first year, which already includes a full scheme of sampling and garment manufacture, there is a very limited timeframe in which the resource tool could be integrated earlier.

The It is also apparent through-testing, stage highlighted the benefit that eonsidering the of valuinge of each sample separately and collectively would benefit the entirety of the range to ensure the resource tool is suitableility for industry and 
educational requirements, and engagement for students. For future development of the In a proposed streamlined set of resources tool, the exclusion of creative samples (such as the knotting samples) could be eliminated, as the results from the testing stage revealed that have outlined that students can explore thesesteh samples with minimal guidelines $_{-} ;$such examples include the knotting samples. During $€ \underline{T}$ The testing stage; also revealed that the majority of students avoided the technical samples were avoided by students-because of the ease of use of many creative alternatives, which required a more ef an-artistic interpretation. In effect, the students found it easier to experiment with the creative samples-were easier to experiment with.

Observations from the experiment over the three weeks outlined potential areas for development which could further contribute to student engagement in sewing. The resources could be further expanded developed-to feature actual garment prototypes. This could potentially engage students further in understanding how each technique could function within a garment.

Although students from the focus group suggested that the standard format for the pilot samples was successful, being flat and of an A5 standard size, it is believed that integrating the techniques into garment toiles would allow students to better appreciate the three-dimensional aspects and application. For example, how seams work on contours, how a fastening might be applied to a centre back, side seam, centre front garmentarea, etc. The resources in effect could become a standard range of bodices, which could feature fastening variations such as different zip, button or placket types in side $\underline{\text { seams, shoulder seams, centre back and centre front areas, with the addition of a range }}$ of seam varieties. 
Future developments of the resources should also take into consideration how the tool could be used by a wider audience. In particular, those who would not necessarily have access to experts to help them to-develop, enhance and evaluate the results of their applications, such as the craft dressmaker community. It is envisaged that to fully address the sewing skills gap, further experimentation would be required and a method for taking the system beyond academia would be necessary. However, it is considered that this initial development of the resource tool is of extreme value.

\section{References}

Beard, C. A., \& Slocum, A. C. (2005). Development of a CAI module and comparison of its effectiveness with traditional classroom instruction. Clothing and Textiles Research Journal, 23, 298-306. doi:10.1177/0887302X0502300410

Crossick, G., \& Greenlees, R. (2014) Crafts Council Publication Studying Crafts:

Trends in Craft Education and Training. Retrieved from: http://www.craftscouncil.org.uk/content/files/Studying_Craft_Report_single_pgs_.pdf

Drapers. (2011). More fighting talk follows SOS debate. Retrieved from:

http://www.drapersonline.com.libaccess.hud.ac.uk/news/save-our-skills/more-fightingtalk-follows-sos-debate/5023871.

Frayling, C. (2011). On Craftsmanship: towards a new Bauhaus. London: Oberon Books. 
Hayes, S., McLoughlin, J., Fairclough, D., \& Cooklin, G. (2012). Cooklin's garment technology for fashion designers (2nd ed.). Chichester: Wiley.

McRobbie, A. (1998). British fashion design: Rag trade or image industry? London: Routledge.

Norum, P. S. (2013). Examination of apparel maintenance skills and practices: Implications for sustainable clothing consumption. Family and Consumer Sciences Research Journal, 42(2), 124-137. doi:10.1111/fcsr.12047

O’Driscoll, M. (2002). Design for manufacture. Journal of Materials Processing Technology, 122, 318-321. Retrieved from http://ac.elscdn.com/S0924013601011323/1-s2.0-S0924013601011323-main.pdf?_tid=4f9db76a$\underline{66 \mathrm{fb}-11 \mathrm{e} 7-82 \mathrm{ea}-}$

$\underline{\text { 00000aacb35e\&acdnat }=1499861701 \_7950 \mathrm{~d} 4 \mathrm{bbed} 4 \mathrm{a} 56110 \mathrm{f} 03417 \mathrm{~b} 53 \mathrm{~d} 62 \mathrm{~b} 70}$

Romeo, L.D., \& Lee, Y-A. (2013). Creative and technical design skills: are college apparel curriculums meeting industry needs? International Journal of Fashion Design, Technology and Education, Vol. 6, 201-209. doi.org/10.1080/17543266.2013.783629

Swift, K. G., \& Brown, N. J. (2003). Implementation strategies for design for manufacture methodologies. Proceedings of the Institution of Mechanical Engineers, Part B: Journal of Engineering Manufacture, 217, 827-833.

doi.org/10.1243/09544050360673198 
